\title{
Analysis of Kalman Filter Based Approaches for Fusing Out-of-Sequence Measurements corrupted by Systematic Errors
}

\author{
Moritz Koplin * and Wilfried Elmenreich ${ }^{\dagger}$
}

\begin{abstract}
This paper relates to systems for target tracking by fusing measurements from heterogeneous sensors. The measurements are corrupted by systematic errors such as offsets in the value domain and the fusion being complicated due to an Out-of-Sequence measurements problem. We present an example where the offsets of measurements from two sensors are equally large and have opposite algebraic signs. We show that the timely concentrated fusion of measurements according to a simple buffering approach is better suited for diminishing the unwanted effects of systematic errors than the scattered fusion which results from immediately fusing Out-of-Sequence measurements by sophisticated Outof-Sequence measurement fusing algorithms.
\end{abstract}

\section{Introduction}

Many papers on Kalman filtering suggest to use the Kalman filter solely in scenarios where the fused measurements are corrupted by zero mean Gaussian distributed errors. However due to the lack of feasible alternatives, the Kalman filter is applied to a wide variety of scenarios including the fusion of measurements that are corrupted by systematic errors such as offsets.

This corruption of measurements by offsets is an often observed problem in ground target tracking using sensors that emit radiation and derive information such as position and speed of a moving object by analyzing the reflected radiation (e.g. radars or laser scanners). Said offsets can be be caused by reflection points mov-

\footnotetext{
${ }^{*}$ M. Koplin (née Mauthner) is with the Technical University of Vienna, 182 Institut fuer technische Informatik, Treitlstraße 3, 1040 Vienna, Austria (e-mail: e0427797@student.tuwien.ac.at)

$\dagger$ W. Elmenreich is with the Alpen-Adria-Universität Klagenfurt, Institut für Vernetzte und Eingebettete Systeme, Universitätsstraße 65-67, 9020 Klagenfurt, Austria (e-mail: Wilfried.Elmenreich@uniklu.ac.at)
}

ing on the surface of an object. As the shape of the object itself can often only roughly be estimated, the change of location of the reflection point on the object's surface can not be modeled.

In order to increase the fusion system robustness against such systematic errors it is feasible to use multiple heterogeneous sensors that will presumably not be affected over a long time period by the same systematic error.

A problem that is often observed in such multisensor fusion systems is that of Out-of-Sequence measurements (OOSMs). The OOSM problem arises as the original Kalman filter algorithm can only deal with chronologically ordered measurements which makes it impossible to fuse OOSMs. In order to handle OOSMs, highly sophisticated Kalman filter based algorithms that can cope with not chronologically ordered measurements (ADVA) have been developed during recent years. A less sophisticated alternative is to use a Kalman filter based buffering approach (BUFF) wherein non-OOSMs are delayed until the awaited OOSM is available.

In order to compare the ADVA approach with the BUFF approach in regard to their robustness to measurements that are corrupted by systematic errors such as offsets in the value domain we analyze an exemplary scenario.

The remainder of this paper is organized as follows. Section 2 presents the simulation environment, i.e. a basic fusion system, the Kalman filter algorithm (for notational consistency only) and the temporal constraints taken into account. Section 3 discusses the OOSM problem and algorithms that have been developed during recent years in order to overcome said problem. Section 3 further defines which OOSM fusing algorithm is used in the simulation environment. Section 4 presents results obtained using the simulation environment for the exemplary scenario and section 5 analyzes said results. The paper is concluded in section 6 . 


\section{Simulation Environment}

In [1-4] Mauthner et al. studied the feasibility of time-triggered sensor fusion with the focus on deriving an optimal temporal and algorithmic parameter set using a simulation environment wherein all assumptions of basic Kalman filtering were met. Therein it is shown that the ADVA approach for fusing OOSMs usually outmatches the BUFF approach. In order to make the papers' results comparable to this paper, the same time-triggered simulation environment dealing with the tracking of objects for use in safety related driver assistance systems as in papers [2-4] is used. This simulation environment is repeated here solely for convenience and notational consistency throughout the paper.

We consider a system with two heterogeneous sensors that measure the kinematic states of surrounding objects and transmit these measurements via a timetriggered bus system to a fusion/prediction core. The fusion/prediction core fuses the incoming measurements and then periodically predicts the fused object states from state time $(S T)$ to real time $(R T)$ in order to provide an update for a safety related application (see figure 1).

The prediction interval $(R T-S T)$ is therefore equal to the time that elapses while a measurement is generated by preprocessing a snapshot of the surrounding environment, transmitted as a message over the bus system to the fusion/prediction core and fused with the object states stored on the fusion/prediction core.

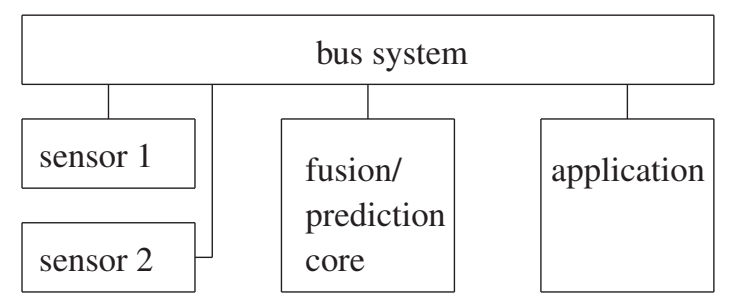

Figure 1. Target tracking system

Figure 2 depicts the timing parameters for a process within our sensor fusion system. We use the following abbreviations in order to describe the timing parameters of all processes:

- the cycle time $t_{C}$ of a process is the time interval that this process requires for completion of its service while the start of two consecutive processes is $t_{C}$ apart

- the execution time $t_{T}$ of a process is the time interval in which this process completes its service

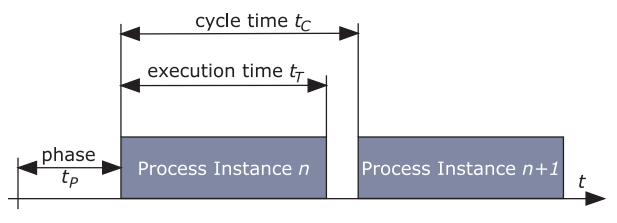

Figure 2. Timing model of a process

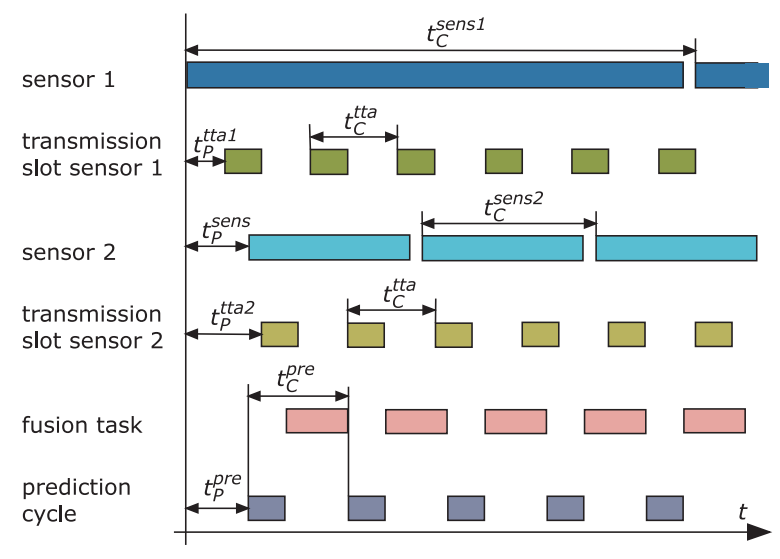

Figure 3. System schedule

where the start of two consecutive processes can be more than $t_{T}$ apart

- the phase $t_{P}$ of a process is defined as the time interval between the start of this process relative to the start of the first cycle of sensor 1

In figure 3 the system schedule is visualized. Therein, time intervals in which a sensor or the fusion/prediction core are active $\left(t_{T}\right)$ are indicated by bars. Furthermore the process cycle times $\left(t_{C}\right)$ and phases $\left(t_{P}\right)$ are indicated for each process. Figure ?? shows all cycle times $t_{C}$, process execution times $t_{T}$ and phases $t_{P}$ (relative to sensor 1$)$, of sensor preprocessing $\left(t_{C}^{\text {sens } 1}, t_{C}^{\text {sens } 2}\right.$, $\left.t_{P}^{\text {sens }}\right)$, measurement transmission $\left(t_{C}^{t t a}, t_{P}^{t t a 1}, t_{P}^{t t a 2}\right)$ and prediction of the application update $\left(t_{C}^{\text {pre }}, t_{T}^{\text {pre }}\right.$ and $\left.t_{P}^{\text {pre }}\right)$ that are assumed to be fixed and are referred to as the system schedule throughout the paper. The fusion process itself which is characterized by the time needed to fuse a measurement $t_{T}^{f u s}$ ( $t_{T}^{f u s / I S}$ for In-Sequence mea-

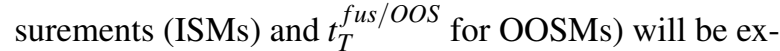
ecuted whenever measurements are available at the fusion/prediction core and resources are available as indicated by the dashed line whose gaps result from the fact that the fusion/prediction core also needs resources for prediction of the application update indicated by the line below said line.

To process the incoming measurements at the fusion/prediction core, we use a Kalman filter as de- 
scribed in [5] and for processing the OOSM we use an ADVA approach as defined at the end of section 3 or an BUFF approach. As the Kalman filter is a widely used and well-known algorithm for tracking moving targets, we will only give a short overview of the notation used throughout this paper.

The estimated state vector of the tracked object $\hat{\vec{x}}\left(t_{k-1} \mid t_{k-1}\right)$ is updated with measurements at discrete points in time $t_{k}$. The state prediction from $t_{k-1}$ to $t_{k}$ is given by $\hat{\vec{x}}\left(t_{k} \mid t_{k-1}\right)=F\left(t_{k}-t_{k-1}\right)$. $\hat{\vec{x}}\left(t_{k-1} \mid t_{k-1}\right)$. The predicted state covariance matrix is computed as $P\left(t_{k} \mid t_{k-1}\right)=F\left(t_{k}-t_{k-1}\right) \cdot P\left(t_{k-1} \mid t_{k-1}\right)$. $\left(F\left(t_{k}-t_{k-1}\right)\right)^{T}+Q_{t_{k-1}}$. The measurement update of the predicted system state is $\hat{\vec{x}}\left(t_{k} \mid t_{k}\right)=\hat{\vec{x}}\left(t_{k} \mid t_{k-1}\right)+K_{t_{k}} \cdot \vec{v}_{t_{k}}$ with $K_{t_{k}}=P\left(t_{k} \mid t_{k-1}\right) \cdot H_{t_{k}}^{T} \cdot\left(H_{t_{k}} P\left(t_{k} \mid t_{k-1}\right) H_{t_{k}}^{T}+R_{t_{k}}\right)^{-1}$, $\vec{v}_{t_{k}}=\vec{z}_{t_{k}}-\hat{\vec{z}}_{t_{k}}$ and $\hat{\vec{z}}_{t_{k}}=H_{t_{k}} \cdot \hat{\vec{x}}\left(t_{k}\right)$. The update of the covariance matrix is given by $P\left(t_{k} \mid t_{k}\right)=\left(I-K_{t_{k}} \cdot H_{t_{k}}\right)$. $P\left(t_{k} \mid t_{k-1}\right) \cdot\left(I-K_{t_{k}} \cdot H_{t_{k}}\right)^{T}+K_{t_{k}} \cdot R_{t_{k}} \cdot K_{t_{k}}^{T}$. The fusion/prediction core processes a measurement within $t_{T}^{f u s}$.

\section{Out-of-Sequence Measurement Treat- ment}

In multi-sensor target tracking systems, measurements of two sensors which provide information about the same object can arrive Out-of-Sequence (OOS) [6, 7], that means not in chronological order. Often, OOS behavior is caused by an indeterministic transmission system, where the transmission time of a message may vary so much that a message from a later measurement may overtake a newer measurement. Such behavior is caused by transmission protocols with many retries or in networks with dynamic routing (Internet, wireless sensor networks).

However, even if communication protocols with deterministic behavior, such as time-triggered approaches like Flexray [8], TTCAN [9], TTP [10], or TTP/A [11] are used, the OOSM problem may arise.

Figure 4 depicts a situation with an OOSM problem that is independent from communication system issues. Due to different preprocessing times the indicated measurement from sensor 2 is received earlier at the fusion/prediction core than the indicated measurement from sensor 1 although the measurement from sensor 2 is more recent.

As the Kalman filter in the form of [5] can not handle OOSMs, architectural and algorithmic solutions have been developed which will be called advanced algorithms (ADVA) throughout this paper.

Please note that $t_{\kappa}$ represents the OOSM time stamp and $t_{k}$ represents the time stamp of the informa-

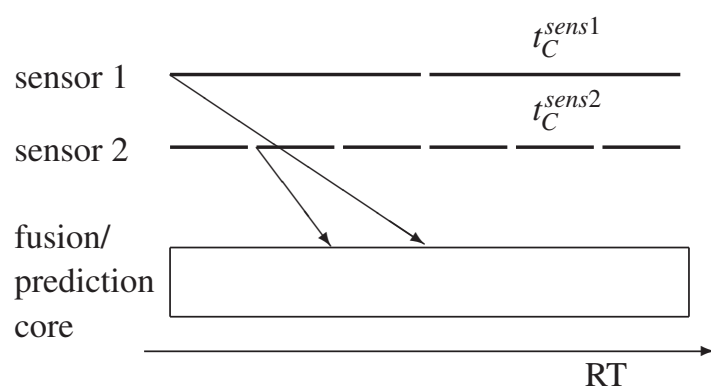

Figure 4. Origin of out-of-sequence measurements

tion which updated the fusion before the OOSM was received.

Larsen et al. present a suboptimal multi-lag filtering algorithm for linear systems [12]. If information is OOS, a correction term derived from the covariance matrices is set up, which is updated every time step. As soon as the delayed information comes up, this correction term is used to update the current state estimate with the delayed information.

Bar-Shalom presents an optimal one-lag tracking algorithm for linear systems [13]. The delayed information is incorporated by computing the update of the state at time $t_{k}$ with the residual of the OOSM and the retrodicted state to the time $\kappa$ as well as the covariance matrices between the states at $t_{k}$ and $t_{\kappa}$. In $[14,15]$ BarShalom et al. extend the presented one-lag algorithm to deal with multi-lag OOSMs by virtually compressing the information of the updates between $t_{\kappa}$ and $t_{k}$ into one update. This approach is further extended to a multi-model approach in [16].

Mallick et al. describe an extension to the algorithm presented in [13] toward a multi-lag, single-model and a one-lag, multi-model approach [17]. In [18], Mallick et al. present a multi-lag, single-model algorithm that includes data association, likelihood computation and hypothesis management. After presenting a particle filter for OOSM processing in [19], Mallick and Marrs compare particle filter (algorithm from [20]) and Kalman filter (algorithm from [21]), based on multi-lag filtering algorithms for linearized systems, in [22].

Orton and Marrs present the incorporation of OOSMs with particle filters [20,23,24].

Zhang describes an algorithm in [21] that is stated to be the general case of [13] and [17]. She further differentiates between globally optimal solutions and solutions optimal for the information given. In [25], she extends the before mentioned algorithms and establishes the connection to the work of Challa and Wang which will be discussed further down. 
Maskel et al. present an approach similar to Zhang by trying to describe the algorithms as specific approximations to an overall framework, being optimal respectively suboptimal for the assumptions made and the information given [26].

Challa and Wang present an augmented state vector, that is a temporally staggered vector, consisting of the present state and past states. This enables the information fusion to incorporate measurements corresponding to past states in an optimal, elegant way but is computationally enormous expensive [27] (optimal multilag filtering algorithm for linear systems). To overcome the computationally expensive augmented state algorithm, Challa and Wang introduce the iterated augmented state algorithm [28]. In [29], they additionally describe the use of these algorithms in scenarios with clutter. Furthermore, Wang and Challa extend their algorithm toward an interacting multiple-model approach in [30].

In [31], Anxi et al. present a unified suboptimal one-lag, multi-lag and mixed-lag advanced algorithms (consider a sequence with two OOSMs 1 and 2 with $t_{\kappa_{1}}<t_{\kappa_{2}}$ and $t_{k_{1}}>t_{k_{2}}$ ).

For sake of discussion we will only consider the OOSM processing algorithm A $l 1$ of [15] referred to as ADVA as it is best suited for the presented exemplary scenario.

\section{Simulation}

In our simulation we track the one-dimensional motion of a target by fusing measurements of two heterogeneous sensors that provide measurements of the $\mathrm{x}$-position of the target. The used system model can be represented by

$$
F\left(t_{k}-t_{k-1}\right)=\left[\begin{array}{cc}
1 & t_{k}-t_{k-1} \\
0 & 1
\end{array}\right]
$$

and

$$
Q_{t_{k-1}}=\left[\begin{array}{ll}
\frac{\left(t_{k}-t_{k-1}\right)^{3}}{3} & \frac{\left(t_{k}-t_{k-1}\right)^{2}}{2} \\
\frac{\left(t_{k}-t_{k-1}\right)^{2}}{2} & t_{k}-t_{k-1}
\end{array}\right] \cdot q
$$

with $q=0.5 \frac{\mathrm{m}^{2}}{\mathrm{~s}^{3}}$ being the power spectral density of the continuous time process noise. The measurements of the two sensors are $z_{t_{k}}^{\text {sens } 1}=v_{0} \cdot t_{k}+o f f s e t^{\text {sens } 1}$ and $z_{t_{k}}^{\text {sens } 2}=v_{0} \cdot t_{k}+o f f s e t^{\text {sens } 2}$ with $v_{0}=2 \frac{m}{s}, o f f s e t^{\text {sens } 1}=2 \mathrm{~m}$ and offset $^{\text {sens } 2}=-2 \mathrm{~m}$. As solely the $\mathrm{x}$-position is measured, the transformation matrices between state space and measurement space are

$$
H_{t_{k}}^{\text {sens } 1 / 2}=\left[\begin{array}{ll}
1 & 0
\end{array}\right]
$$

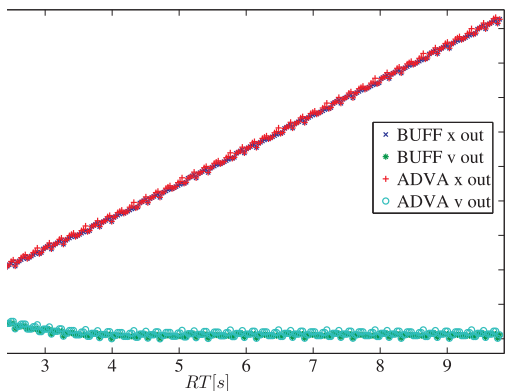

Figure 5. Overview: real time x-position (x-out) and speed (v-out) estimated by ADVA respectively BUFF approach with offset ${ }^{\text {sens } 1}=2 \mathrm{~m}$ and offset ${ }^{\text {sens } 2}=-2 \mathbf{m}$

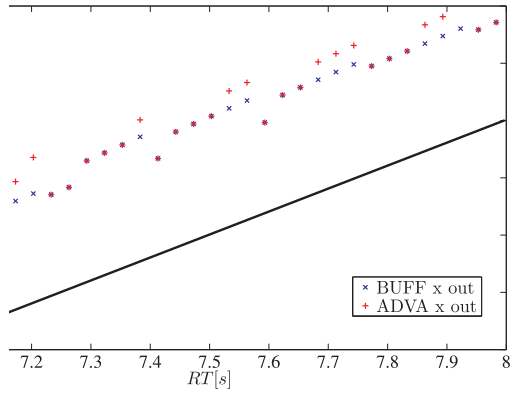

Figure 6. Close-up: real time x-position (x out) estimated by ADVA respectively BUFF approach with offset ${ }^{\text {sens } 1}=2 \mathbf{m}$ and $o f f s e t^{\text {sens } 2}=-2 \mathbf{m}$

The measurement noise of both sensors is characterized by their variances

$$
R_{t_{k}}^{\text {sens } 1 / 2}=1 m^{2}
$$

The temporal evolution of the simulation environment is defined by $t_{C}^{\text {sens } 1}=180 \mathrm{~ms}, t_{C}^{\text {sens } 2}=80 \mathrm{~ms}, t_{C}^{t t a}=$ $5 \mathrm{~ms}, t_{T}^{t t a 1}=1 \mathrm{~ms}, t_{P}^{t t a 1}=1 \mathrm{~ms}, t_{P}^{\text {sens }}=0 \mathrm{~ms}, t_{P}^{\text {sens }}=0 \mathrm{~ms}$, $t_{P \text { pre }}^{t t a 2}=2 \mathrm{~ms}, t_{T}^{\text {tta2 }}=1 \mathrm{~ms}, t_{T}^{\text {fus } / I S}=5 \mathrm{~ms}, t_{T}^{\text {fus } / O O S}=8 \mathrm{~ms}$, $t_{C}^{\text {pre }}=30 \mathrm{~ms}, t_{T}^{\text {pre }}=2 \mathrm{~ms}, t_{P}^{\text {pre }}=0 \mathrm{~ms}$

Figure 5 depicts the fusion results for $\mathrm{x}$-position and speed of the target which have been predicted to RT for the ADVA and the BUFF approach. When regarding a close-up of said results as depicted in figure 6 and figure 7 the estimated x-position and the estimated speed of the target, it is obvious that the ADVA approach applied to OOSMs achieves a weaker result than the BUFF approach as the average speed and the average $\mathrm{x}$-position of the target in the ADVA approach is further away from the real values indicated by the straight lines than in the BUFF approach. 


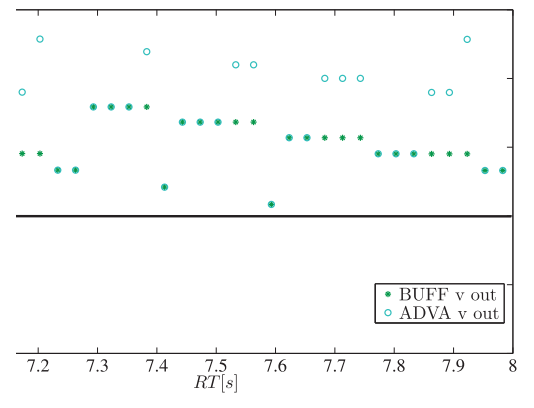

Figure 7. Close-up: real time speed (v out) estimated by ADVA respectively BUFF approach with offset ${ }^{\text {sens } 1}=2 \mathbf{m}$ and $o f f s e t^{\text {sens } 2}=-2 \mathbf{m}$

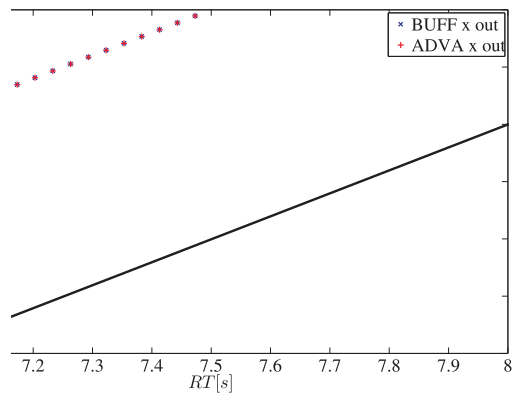

Figure 8. Close-up: real time x-position (x out) estimated by ADVA respectively BUFF approach with offset ${ }^{\text {sens } 1}=2 \mathrm{~m}$ and offset $^{\text {sens } 2}=2 \mathrm{~m}$

Please note that when both offsets have the same algebraic sign and the same numeric values (e.g. offset $^{\text {sens } 1}=2 \mathrm{~m}$ and offset ${ }^{\text {sens } 2}=2 \mathrm{~m}$ ) the performance of the fusion is for the given scenario independent from the chosen OOSM processing approach as can be seen in figure 8 .

\section{Discussion}

The reason for the effect shown in figures 5 to 7 can be understood when recognizing the temporal evolution of the state vector as depicted in figure 9. In the ADVA approach the fusion of measurements is evenly spread over RT (indicated by an arrow) whereas the fusion of measurements in the BUFF approach is concentrated around the time instant (indicated by an ellipse) where the OOSM arrives at the fusion/prediction core. This concentration has the effect that a degradation of the fusion result due to an not modeled systematic error might soon after its generation be diminished by either measurements without this error or even compensated by measurements that suffer the same effect but have an

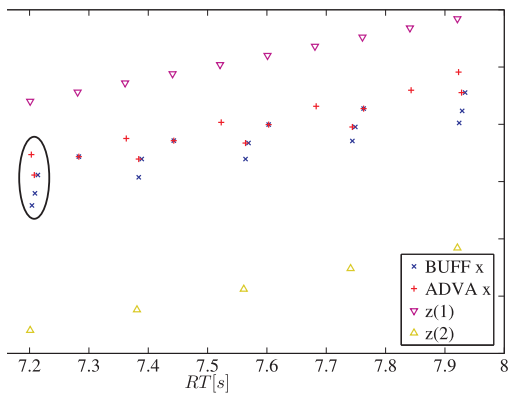

Figure 9. Close up: state time x-position (x) estimated by ADVA respectively BUFF approach and measured $\mathrm{x}$-position ( $\mathrm{z}$ )

opposite algebraic sign as the offsets in our example.

This concentration of fusion steps around certain instants is advantageous as it minimizes the time when the filter state is heavily corrupted.

\section{Conclusions}

In this paper we have presented an example for a Kalman filter based fusion system which is supplied with measurements being corrupted by systematic errors such as offsets in the value domain. We have shown that buffering the ISM measurements achieves better results than fusing the OOSM with sophisticated OOSM algorithms. We believe that this example is part of a class of target tracking systems which employ multiple heterogeneous sensors where the concentration of fusing measurements around given instants in time is most suitable for exploiting measurement redundancy.

\section{References}

[1] M. Mauthner, W. Elmenreich, A. Kirchner, and D. Boesel, "Out-of-sequence measurements treatment in sensor fusion applications: Buffering versus advanced algorithms," Proceedings of the 4. Workshop Fahrerassistenzsysteme, pp. 20-30, October 2006, loewenstein/Hoesslinsuelz, Germany.

[2] M. Mauthner, "Out-of-sequence measurements treatment in sensor fusion applications: Optimal sensor fusion speed and synchronization," Proceedings of the 4th International Workshop on Intelligent Transportation (WIT 2007), March 2007, hamburg, Germany.

[3] M. Mauthner, R. Altendorfer, W. Elmenreich, and A. Kirchner, "Optimization of sensor, bus, and fusion schedules of a time-triggered sensor fusion system," Proceedings of the IEEE Intelligent Vehicles Symposium, June 2007, istanbul, Turkey.

[4] M. Mauthner, W. Elmenreich, and A. Kirchner, "Anal- 
ysis of sensor and fusion schedules of a time-triggered sensor fusion system," in Proceedings of the Tenth International Conference on Information Fusion. Québec, Canada: ISIF, Jul. 2007.

[5] A. Gelb, J. Kasper, R. Nash, C. Price, and A. Sutherland, Applied Optimal Estimation, A. Gelb, Ed. The M.I.T. Press, 1974.

[6] S. Thomopoulos and L. Zhang, "Distributed filtering with random sampling and delay," Proceedings on the 27th Conference on Decision and Control, December 1988.

[7] Y. Bar-Shalom, Multitarget-Multisensor Tracking: Applications and Advances, ser. Multitarget-Multisensor Tracking. Artech House, 2000, vol. 3.

[8] FlexRay Consortium, "Flexray communications system," Protocol Specification, Dec. 2005, version 2.1 Revision A.

[9] F. Hartwich, B. Müller, T. Führer, and R. Hugel, "Time triggered communication on CAN," in Proceedings 7th International CAN Conference, Amsterdam, The Nederlands, 2000.

[10] TTAGroup, Specification of the TTP/C Protocol. TTAGroup, 2003, available at www.ttagroup.org.

[11] H. Kopetz et al., "Specification of the TTP/A protocol," Vienna University of Technology, Vienna, Austria, Research Report 61/2002, Sep. 2002, version 2.00.

[12] T. D. Larsen, N. K. Poulsen, N. A. Andersen, and O. Ravn, "Incorporation of time delayed measurements in a discrete-time kalman filter," Proceedings of the 37th Conference on Decision and Control, Tampa, Florida, USA, pp. 3972-3977, 1998.

[13] Y. Bar-Shalom, "Update with out-of-sequence measurements in tracking: Exact solution," in IEEE Transactions on Aerospace and Electronic Systems, vol. 38, no. 3. IEEE - Institute of Electrical and Electronics Engineers, Inc., Jan. 2002, pp. 769-778.

[14] Y. Bar-Shalom, M. Mallick, H. Chen, and R. Washburn, "One-step solution for the general out-of-sequencemeasurement problem in tracking," Proceedings of the 2002 IEEE Aerospace Conference, March 2002.

[15] Y. Bar-Shalom, H. Chen, and M. Mallick, "One-step solution for the multistep out-of-sequence-measurement problem in tracking," IEEE Transactions on Aerospace and Electronic Systems, vol. 40, no. 1, January 2004.

[16] Y. Bar-Shalom and H. Chen, "IMM estimator with out-of-sequence measurements," IEEE Transactions on Aerospace and Electronic Systems, vol. 41, no. 1, January 2005.

[17] M. Mallick, S. Coraluppi, and C. Carthel, "Advances in asynchronous and decentralized estimation," Proc. 2001 IEEE Aerospace Conference, vol. 4, pp. pages 18731888, March 2001, big Sky MT.

[18] M. Mallick, J. Krant, and Y. Bar-Shalom, "Multi-sensor multi-target tracking using out-of-sequence measurements," in Proceedings of the Fifth International Conference on Information Fusion, vol. 1. IEEE - Institute of Electrical and Electronics Engineers, Inc., Jul. 2002, pp. 135-142.
[19] M. Mallick, T. Kirubarajan, and S. Arulampalam, "Out-of-sequence measurement processing for tracking ground target using particle filters," Aerospace Conference Proceedings IEEE, vol. 4, pp. 1809-1818, 2002.

[20] M. Orton and A. Marrs, "Incorporation of out-ofsequence measurements in non-linear dynamic systems using particle filters," University of Cambridge: Department of Engineering, Cambridge, UK, Technical Report $4511,2001$.

[21] K.-S. Zhang, X. R. Li, and Y.-M. Zhu, "Optimal update with out-of-sequence measurements for distributed filtering," in Proc. 2002 International Conference on Information Fusion, Annapolis, MD, July 2002, July 2002, pp. 1519-1526.

[22] M. Mallick and A. Marrs, "Comparison of the KF and particle filter based out-of-sequence measurement filtering algorithms," 6th International Conference on Information Fusion, pp. 422-430, 2003.

[23] M. Orton and A. Marrs, "A bayesian approach to multitarget tracking and data fusion with out-of-sequence measurements," Target Tracking: Algorithms and Applications, vol. 1, pp. 15/1- 15/5, October 2001.

[24] M. Orton and A. Marrs, "Particle filters for tracking with out-of-sequence measurements," IEEE Transactions on Aerospace and Electronic Systems, vol. 41, no. 2, 2005.

[25] K.-S. Zhang, X. R. Li, and Y.-M. Zhu, "Optimal update with out-of-sequence measurements," IEEE Transactions on Signal Processing, vol. 53, no. 6, JUNE 2005.

[26] S. Maskell, R. Everitt, R. Wright, and M. Briers, "Multi target out-of-sequence data association," Conference of Information Fusion, 2004.

[27] S. Challa, J. Legg, and X. Wang, "Track-to-track fusion of out-of-sequence tracks," Proceedings of the Fifth International Conference on Information Fusion, vol. 2, pp. pages 919- 926, 2002.

[28] S. Challa, R. Evans, X. Wang, and J. Legg, "A fixedlag smoothing solution to out-of-sequence information fusion problems," Communications in Information and Systems, vol. 2, no. 4, pp. 325-348, December 2002.

[29] S. Challa, R. Evans, and X. Wang, "A bayesian solution and its approximations to out-of-sequence measurement problems," Information Fusion, vol. 4, no. 3, pp. 185199, 2003.

[30] X. Wang and S. Challa, "Augmented state IMM-PDA for OOSM solution to maneuvering target tracking in clutter," Proceedings of Radar 2003 International Conference, pp. 479-485, September 2003, adelaide, Australia.

[31] Y. Anxi, L. Diannong, H. Weidong, and D. Zhen, "A unified out-of-sequence measurements filter," IEEE International Radar Conference, pp. 453 - 458, May 2005. 